\title{
An Unusual Case of Gastric Gnathostomiasis Caused by Gnathostoma spinigerum Confirmed by Video Gastroscopy and Morphological and Molecular Identification
}

\author{
Kookwan Sawadpanich, ${ }^{1}$ Nitiwat Chansuk, ${ }^{2}$ Patcharaporn Boonroumkaew, ${ }^{3,4}$ Lakkhana Sadaow, ${ }^{3,4}$ Rutchanee Rodpai, ${ }^{3,4}$ \\ Oranuch Sanpool, ${ }^{3,4}$ Penchom Janwan, ${ }^{5}$ Pewpan M. Intapan, ${ }^{3,4}$ and Wanchai Maleewong ${ }^{3,4 *}$ \\ ${ }^{1}$ Gastroenterology and Hepatology Division, Department of Medicine, Faculty of Medicine, Khon Kaen University, Khon Kaen, Thailand; ${ }^{2}$ Internal \\ Medicine Unit, Ratchaphruek Hospital, Khon Kaen, Thailand; ${ }^{3}$ Department of Parasitology, Faculty of Medicine, Khon Kaen University, Khon Kaen, \\ Thailand; ${ }^{4}$ Mekong Health Science Research Institute, Khon Kaen University, Khon Kaen, Thailand; ${ }^{5}$ Department of Medical Technology, School of \\ Allied Health Sciences, Walailak University, Nakhon Si Thammarat, Thailand
}

\begin{abstract}
Human gnathostomiasis is a harmful foodborne parasitic infection caused by nematodes of the genus Gnathostoma. Here, we report an unusual case of gastric gnathostomiasis seen in a hospital in Thailand along with the clinical characteristics, treatment, and outcome. A 39-year-old man presented with complaints of epigastric pain, dizziness, and history of passing dark, tarry stools for 2 days. The patient had a history of consuming raw freshwater fish. Supplementary differential diagnosis was performed via rapid serological testing, and presence of the causative agent was confirmed based on video gastroscopy, morphology of the removed parasite, and molecular identification. After its surgical removal from the stomach, the parasite was morphologically identified as Gnathostoma species. Molecular identification was performed via DNA extraction from the recovered worm, and amplification and sequencing of the second internal transcribed spacer (ITS2) region and partial cytochrome $c$ oxidase subunit I (cox1) gene. The ITS2 and cox1 sequences were consistent with those of Gnathostoma spinigerum. Clinicians in endemic areas should therefore be aware of the rare clinical manifestations and use of supplementary serological tests to facilitate early diagnosis and treatment of gastric gnathostomiasis.
\end{abstract}

Human gnathostomiasis is a harmful emerging infectious disease caused by the foodborne spirurid worm Gnathostoma spp. (Nematoda: Gnathostomatidae). ${ }^{1-3}$ At least six species, including Gnathostoma spinigerum, Gnathostoma doloresi, Gnathostoma hispidum, Gnathostoma nipponicum, Gnathostoma malaysiae, ${ }^{4}$ and Gnathostoma binucleatum, are reported to cause human diseases, the most common in Asia being G. spinigerum. ${ }^{3}$ Humans become an accidental host through consumption of undercooked freshwater fish or meat containing infective larvae. The worms cannot mature in the human body, and if they are removed in the early stages of infection, they often are in the advanced third larval stage. However, in some cases where the parasites remain within the human body for an extended period, development may proceed to the fourth larval stage or growth into immature adults. ${ }^{5-8}$ The larvae migrate into various subcutaneous tissues and organs, including the central nervous system and eyes, and these exert severe pathologic effects leading to serious complications or death. ${ }^{9}$ Clinical manifestations are generally divided into two major disease categories, specifically, cutaneous gnathostomiasis and visceral gnathostomiasis. ${ }^{7,10}$ Cutaneous gnathostomiasis caused by worm migration into subcutaneous tissues results in intermittent painful and pruritic migratory swelling; visceral gnathostomiasis because of worm migration into visceral organs often causes eosinophilic abscess and substantial damage. ${ }^{7,10}$ Rare cases of intraabdominal gnathostomiasis have additionally been documented. In 1980, Daengsvang ${ }^{9}$ described five cases requiring removal of immature worms from the intraabdomen near the stomach and ileocecal area (four cases) or cecum wall (one case). Seguchi et al. ${ }^{11}$ reported a gnathostomiasis case caused by advanced third-stage larvae of

*Address correspondence to Wanchai Maleewong, Department of Parasitology, Faculty of Medicine, Khon Kaen University, Khon Kaen, Thailand. E-mail: wanch_ma@kku.ac.th
G. doloresi in the colonic ileus near the hepatic flexure. Here we report the first confirmed case of gastric gnathostomiasis caused by an immature male $G$. spinigerum based on serology, video gastroscopy, and morphological and molecular identification of the parasite source.

A 39-year-old male patient presented at the emergency department complaining of epigastric pain and had passed dark, tarry stools for 2 days, along with episodes of dizziness for 2 months prior to hospital admission. The patient had a history of consuming raw freshwater fish (known locally as koi-pla). He denied any regular drug use and had no known history of disease. Physical examination revealed pale conjunctivae, but no remarkable features in other organs. Hematocrit level was $15 \%$. The patient received a blood transfusion (4 units) for anemia. Video gastroscopy revealed a gastric ulcer measuring $2 \times 2 \mathrm{~cm}$ with an adherent clot in the body of the stomach and movement caused by a parasitic roundworm (Supplemental video). The roundworm was removed using biopsy forceps and morphologically identified as $G$. spinigerum-like based on the characteristics of the body spines and cephalic hooklets, number of hooklets, and number of hooklet rows on the head bulb under a stereomicroscope. ${ }^{9}$ The total body size was $3 \mathrm{~mm}$ in width and $7 \mathrm{~mm}$ in length. The body was covered with cuticular spines and comprised eight rows of hooklets on the head bulb (Figure 1). Histological analyses revealed acute gastritis and ulceration with marked eosinophilic infiltration. Immunochromatography with the KAN gnathostomiasis kit (Adtec Inc., Oita, Japan) disclosed positivity for serum immunoglobulin $\mathrm{G}$ against $\mathrm{G}$. spinigerum (Figure 2). ${ }^{12}$

The recovered immature worm was kept in $70 \%$ ethanol at $-70^{\circ} \mathrm{C}$ at Khon Kaen University Srinagarind Hospital until DNA extraction. The patient made a full recovery after extraction of the parasite.

The study protocol was approved by the Khon Kaen University Ethics Committee for Human Research (HE621234) and was based on the relevant guidelines and regulations of 


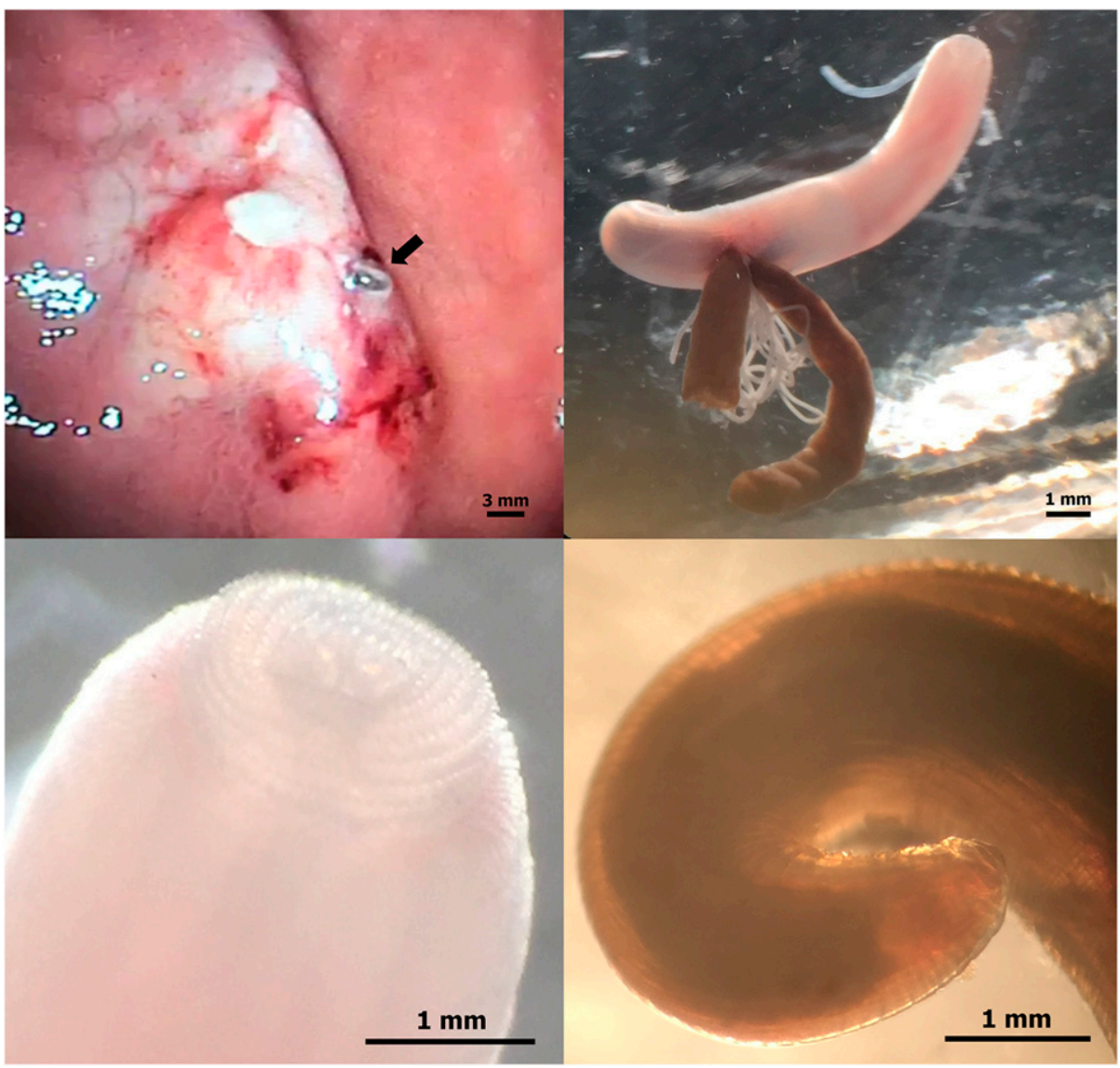

FIGURE 1. Photomicrographs of immature male Gnathostoma spinigerum perforating the gastric wall (upper left, black arrow indicates the parasite), recovered immature adult male (upper right), head with eight cephalic rows (lower left), and tail with pseudo-bursa (lower right). This figure appears in color at www.ajtmh.org.

the Ethical Principles for Medical Research Involving Human Subjects outlined in the Declaration of Helsinki. Genomic DNA from the immature male worm was extracted using the NucleoSpin ${ }^{\circledR}$ tissue kit (Macherey-Nagel GmbH \& Co. KG, Duren, Germany) according to the manufacturer's instructions. Polymerase chain reaction (PCR) was conducted to amplify the internal transcribed spacer 2 (ITS2) region (partial $5.8 \mathrm{~S}$, entire ITS2, and partial $28 \mathrm{~S}$ regions) with the primer pair NEWS2 and ITS2-RIXO ${ }^{13}$ and two regions of the mitochondrial cytochrome $c$ oxidase subunit I (cox1) gene with two primer pairs (Set 1: GsCox1-1F and GsCox1-1R; Set 2: GsCox1-2F and GsCox1-2R). ${ }^{14}$ Amplification of extracted DNA $(18 \mathrm{ng} / 2.5 \mu \mathrm{L})$ was performed as described previously. ${ }^{14} \mathrm{PCR}$ products were electrophoresed on a $1.0 \%$ $(w / v)$ agarose gel and stained with ethidium bromide to visualize amplified fragments under ultraviolet illumination. Direct sequencing was performed on amplified products in both directions with PCR primers as the sequencing primers using Applied Biosystems $3730 \times$ I DNA Analyzer and ABI Big Dye Version 3.1 (Applied Biosystems, Foster City, CA) by Apical Scientific Sdn Bhd (Selangor, Malaysia).

Sequences were identified and compared using the Basic Local Alignment Search Tool provided by the National Center for Biotechnology Information (U.S. National Library of Medicine, Bethesda, MD; http://blast.ncbi.nlm.nih.gov/Blast.cgi). The sequence alignment was compared with those of known Gnathostoma species in GenBank using the multiple sequence alignment program ClustalW in BioEdit Sequence Alignment Editor, version 7.2.6.1, ${ }^{15}$ and consequently identified as G. spinigerum. The ITS2 region displayed $100 \%$ identity $(100 \%$ coverage) with corresponding regions of $G$. spinigerum from Myanmar, Cambodia, Lao PDR, United States (from imported Asian swamp eels), Indonesia, and Thailand (GenBank accession numbers MK028971, MK028963, MK028960, KF648553, JN408321, and AB181155). The partial mitochondrial cox1 gene sequence exhibited $98.9-99.9 \%$ similarity (100\% coverage) with that of G. spinigerum from Myanmar, Cambodia, China, and Lao PDR (GenBank accession numbers MK033987, MK033974, KP410547, and MK033968). The sequences reported in this study for the ITS2 region and cox1 gene are deposited in the GenBank database under accession numbers MT994563 and MT998288, respectively.

Phylogenetic trees were created using MEGA $X$ software. ${ }^{16}$ The evolutionary of the ITS2 region (605 bp) was inferred using the maximum likelihood method and the bestfit substitution Kimura 2-parameter (K2) model, with nonuniformity of evolutionary rates among sites using a distinct gamma distribution $(+G)$ (Figure $3 A)$. The evolutionary of the partial cox1 gene (1470 bp) was inferred using the maximum likelihood method and the best-fit substitution Tamura-Nei 


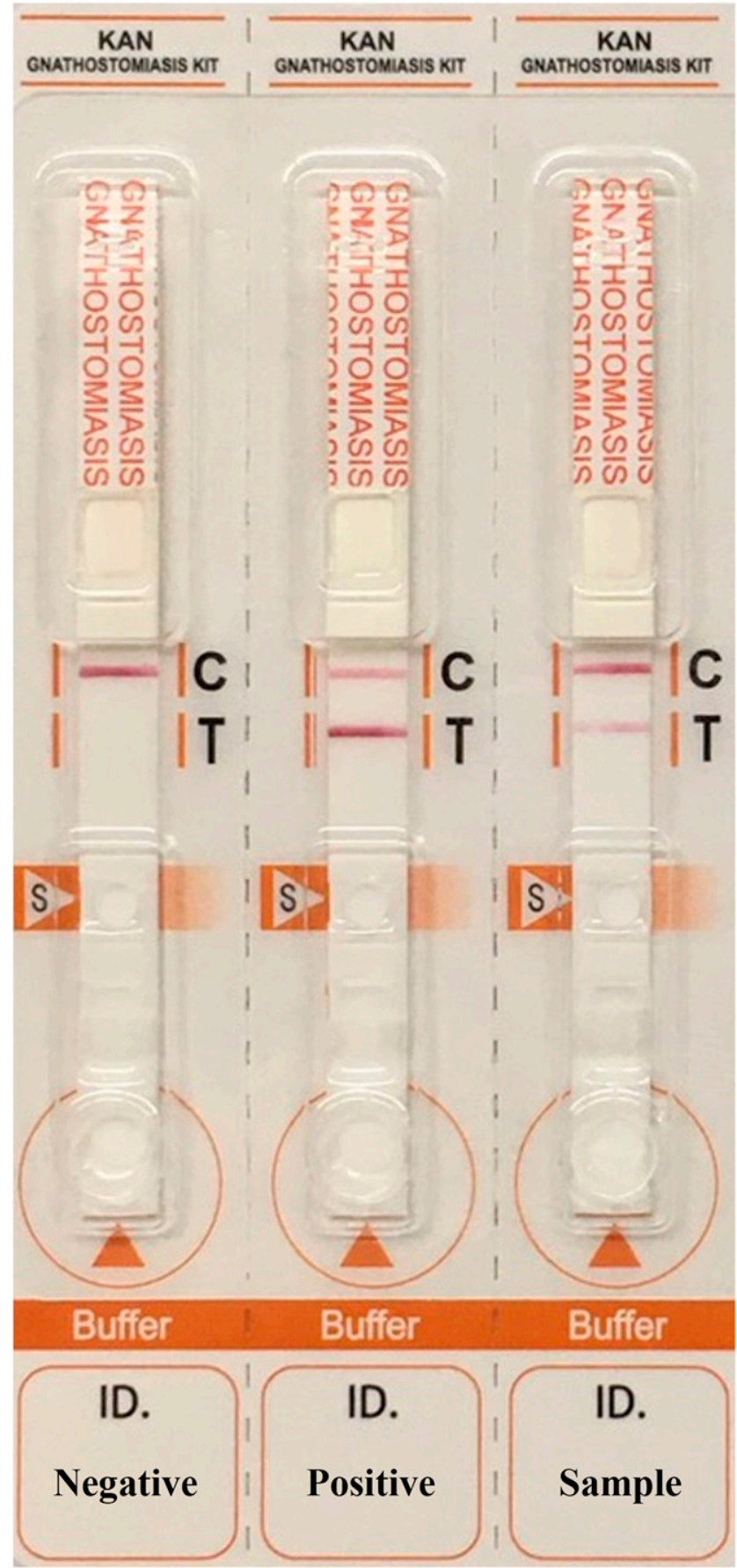

FIGURE 2. Results of the immunochromatographic assay using a KAN gnathostomiasis kit for diagnosis of human gnathostomiasis in negative control (left), positive control (middle), and patient sample sera (right). In a positive serum sample, the test $(T)$ and control (C) lines turn red, whereas only the control line turns red if the serum sample is negative. This figure appears in color at www.ajtmh.org.

(TN93) model, with non-uniformity of evolutionary rates among sites using a distinct gamma distribution $(+G)$ (Figure 3B). Bootstrap percentages were estimated based on 1000 replications. Phylogenetic analysis of the Gnathostoma parasites revealed monophyly of the genus, with each species forming a distinct lineage (Figure 3 ).

In this report, we present an unusual case of gastric gnathostomiasis caused by an immature male G. spinigerum.
The parasite was recovered from the gastric wall, indicating a long duration of incubation after $G$. spinigerum infection, with development to infective third-stage larva. However, the specific routes of infection and larval migration require further investigation. Our patient complained of gastric ulcer, epigastric pain, and passing of dark and tarry stools potentially caused by the invading parasite. Supplementary differential diagnosis of gnathostomiasis was made based on rapid serological testing $^{12}$ and presence of the causative parasite confirmed via video gastroscopy and morphological and molecular identification. Clinicians in endemic areas of gnathostomiasis should therefore be alert to the rare clinical manifestations of gastric gnathostomiasis to facilitate early diagnosis and effective treatment.

Received January 7, 2021. Accepted for publication March 5, 2021.

Published online April 26, 2021.

Note: Supplemental video appears at www.ajtmh.org.

Acknowledgment: We thank Dr. Dylan Southard for language editing of this manuscript through the Khon Kaen University Publication Clinic (Thailand).

Financial support: This study was supported by a Distinguished Research Professor Grant from the Thailand Research Fund [Grant no. DPG6280002], a scholarship under Industrial Post-doctorate Development for Agriculture, Food, Energy and Bio-materials for Future from Khon Kaen University, Thailand [PJ, grant number KKU-PMU-B 63-001], and grants from the Khon Kaen University Research and Graduate studies [RP64010] and Faculty of Medicine [WM and OS, grant numbers DR63101and RG63301]. The fund providers had no role in study design, data collection/interpretation, or the decision to submit the work for publication.

Authors' addresses: Kookwan Sawadpanich, Gastroenterology and Hepatology Division, Department of Medicine, Faculty of Medicine, Khon Kaen University, Khon Kaen, Thailand, E-mail: kookwans@ yahoo.com. Nitiwat Chansuk, Internal Medicine Unit, Ratchaphruek Hospital, Khon Kaen, Thailand, E-mail: nitiwat_internist@hotmail.com. Patcharaporn Boonroumkaew, Lakkhana Sadaow, Rutchanee Rodapai, Oranuch Sanpool, Pewpan M. Intapan, and Wanchai Maleewong, Department of Parasitology, Faculty of Medicine, Khon Kaen University, Khon Kaen, Thailand, E-mails: patcharaporn.bo@kkumail.com, sadaow1986@gmail.com, rutchanee5020@gmail.com, sanpoolor@ yahoo.com, pewpan@kku.ac.th, and wanch_ma@kku.ac.th. Penchom Janwan, Department of Medical Technology, School of Allied Health Sciences, Walailak University, Nakhon Si Thammarat, Thailand, E-mail: pair.wu@gmail.com.

\section{REFERENCES}

1. Moore DAJ, McCroddan J, Dekumyoy P, Chiodini PL, 2003. Gnathostomiasis: an emerging imported disease. Emerg Infect Dis 9: 647-650.

2. Bravo F, Gontijo B, 2018. Gnathostomiasis: an emerging infectious disease relevant to all dermatologists. An Bras Dermatol 93: 172-180.

3. Sanpool O, Intapan PM, Blair D, Nawa Y, Maleewong W, 2018. Gnathostoma. Liu D, ed. Handbook of Foodborne Diseases. Boca Raton, FL: CRC Press, Taylor \& Francis Group, 859-869.

4. Nomura Y, Nagakura K, Kagei N, Tsutsumi Y, Araki K, Sugawara M, 2000. Gnathostomiasis possibly caused by Gnathostoma malaysiae. Tokai J Exp Clin Med 25: 1-6.

5. Maleewong W, Intapan PM, Khempila J, Wongwajana S, Wongkham C, Morakote N, 1995. Gnathostoma spinigerum: growth and development of third-stage larvae in vitro. J Parasitol 81: 800-803. 
A

KF648534 Gnathostoma spinigerum USA

KP784332 Gnathostoma spinigerum LAO

KP941025 Gnathostoma spinigerum THA

KP941024 Gnathostoma spinigerum THA

KP941023 Gnathostoma spinigerum THA

AB181155 Gnathostoma spinigerum THA

KF648553 Gnathostoma spinigerum USA

MK028960 Gnathostoma spinigerum LAO

99 MK028963 Gnathostoma spinigerum KHM

MT994563 Gnathostoma spinigerum THA

MK028971 Gnathostoma spinigerum MMR

-JN408318 Gnathostoma spinigerum IDN

KP784341 Gnathostoma spinigerum THA

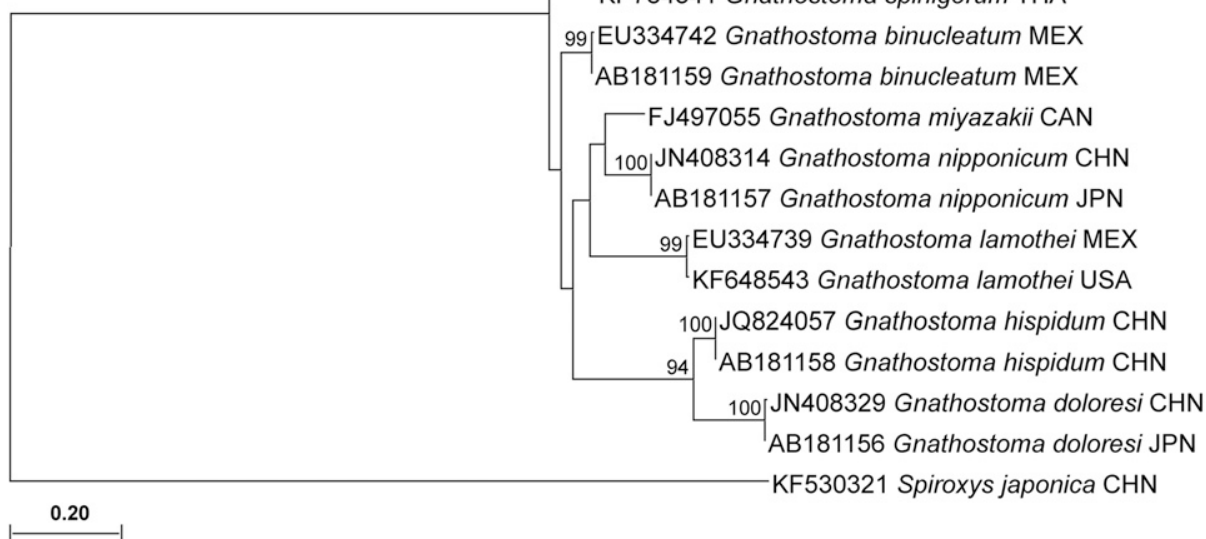

B

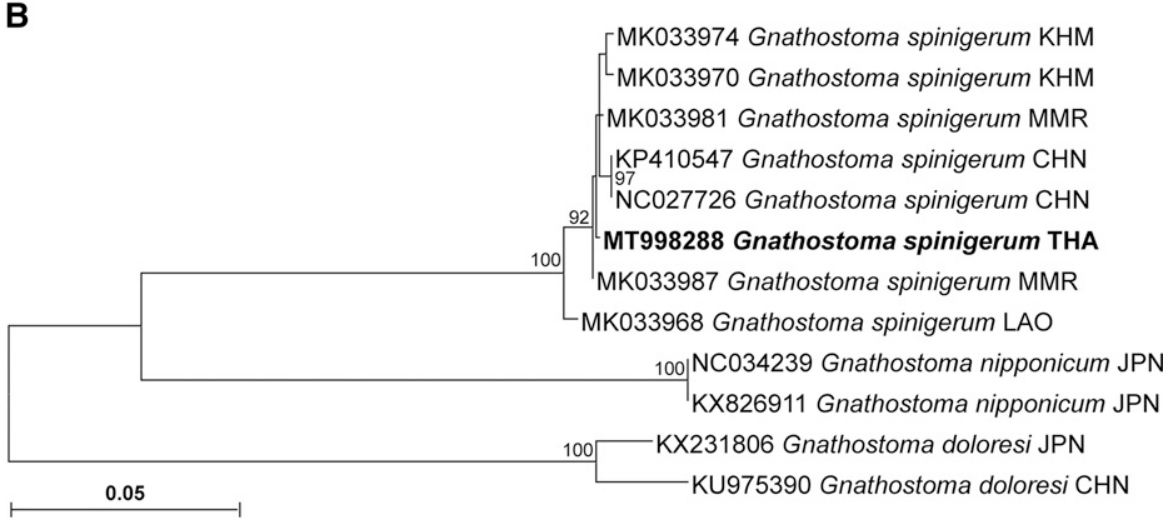

FIGURE 3. Phylogenetic trees of roundworm in the genus Gnathostoma reconstructed using the maximum likelihood method based on the internal transcribed spacer 2 (ITS2) region (A) and partial cytochrome c oxidase subunit I (cox1) gene (B). Gnathostoma species obtained from GenBank are indicated with accession numbers and country codes (ISO 3166-1 alpha-3). Gnathostoma spinigerum sequences obtained from this study are presented in bold (MT994563 for ITS2 and MT998288 for cox1). Bootstrap scores, expressed as a percentage based on 1,000 replications, are indicated at each node. Scale bars indicate substitutions per nucleotide position.

6. Ando K, 2003. Gnathostoma japonicum. Kamegai N, Otsuru $\mathrm{M}$, Hayashi S, eds. Progress in Medical Parasitology, Japan. Vol. 8. Tokyo, Japan: Meguro Parasite Museum, 507-518.

7. Nawa $Y$, Intapan PM, Maleewong W, Camacho SP, 2015. Gnathostoma. Xiao L, Ryan U, Feng Y, eds. Biology of Foodborne Parasites. Boca Raton, FL: CRC Press, Taylor \& Francis Group, 405-426.

8. Phetsouvanh R, Habe $S$, Newton $P$, Vongsouvaht $M$, Horii $Y$, Doanh PN, Nawa Y, 2018. Spontaneous emergence of a Gnathostoma spinigerum adult worm from the abdominal skin: a Laotian woman: a case report. Southeast Asian J Trop Med Public Health 49: 1-5.
9. Daengsvang S, 1980. Monograph on the genus Gnathostoma and gnathostomiasis in Thailand. Tokyo, Japan: SEAMIC Publication No. 21.

10. Waikagul J, Diaz Chamacho SP, 2007. Gnathostomiasis. Murrell KD, Fried B, eds. Food-borne Parasitic Zoonoses. World Class Parasites, Vol. 11. New York, NY: Springer, 235-261.

11. Seguchi K, Matsuno M, Kataoka $H$, Kobayashi T, Maruyama $H$, Itoh H, Koono M, Nawa Y, 1995. A case report of colonic ileus due to eosinophilic nodular lesions caused by Gnathostoma doloresi infection. Am J Trop Med Hyg 53: 263-266.

12. Janwan $P$ et al., 2016. Development and usefulness of an immunochromatographic device to detect antibodies for 
rapid diagnosis of human gnathostomiasis. Parasit Vectors 9: 14.

13. Almeyda-Artigas RJ, Bargues MD, Mas-Coma S, 2000. ITS-2 rDNA sequencing of Gnathostoma species (Nematoda) and elucidation of the species causing human gnathostomiasis in the Americas. J Parasitol 86: 537-544.

14. Boonroumkaew $P$ et al., 2019. Molecular identification and genetic diversity of Gnathostoma spinigerum larvae in freshwater fishes in southern Lao PDR, Cambodia, and Myanmar. Parasitol Res 118: 1465-1472.

15. Hall TA, 1999. BioEdit: a user-friendly biological sequence alignment editor and analysis program for windows 95/98/NT. Nucleic Acids Symp Ser 41: 95-98.

16. Kumar S, Stecher G, Li M, Knyaz C, Tamura K, 2018. MEGA $X:$ Molecular Evolutionary Genetics Analysis across computing platforms. Molecular Mol Biol Evol 35: 1547-1549. 